\title{
Produktionsauftrag: zivilgesellschaftlicher Mehrwert
}

\section{Ökonomisierungsdruck, Organisationsentwicklung und bürgerschaftliches Engagement in der Freien Wohlfahrtspflege}

Rainer Sprengel

Dr. Rainer Sprengel ist wissenschaftlicher Mitarbeiter am Internationalen Graduiertenkolleg »Formenwandel der Bürgergesellschaft « Halle-Tôkyô an der Martin-Luther-Universität HalleWittenberg.

E-Mail rainersprengel@cityweb.de
Die Wiederentdeckung zivilgesellschaftlicher Selbstorganisation und die Verherrlichung des Marktes stellen für die Einrichtungen und Verbände der Freien Woblfahrtspflege einander widersprechende Anforderungen dar. Beantwortet werden kann der Konflikt mit der Rückbesinnung auf die Kernkompetenz der freigemeinnützigen Wohlfahrtspflege: die Ermöglichung und Organisation bürgerschaftlichen Engagements.

In einer modernen Organisationsgesellschaft hängt die Reform- und Innovationsfähigkeit von Gesellschaft und Staat wesentlich von der Fähigkeit der Organisationen $\mathrm{ab}$, sich zu erneuern und weiter zu entwickeln. Das gilt nicht nur für Staat und Wirtschaft, sondern auch für den gesamten Bereich der gemeinwohlorientierten Verbände, Vereine und Stiftungen, die den Dritten Sektor (Non-Profit-Bereich) neben Staat und Markt bilden.

Wie dramatisch die Wandlungen in den letzten dreißig Jahren waren, kann man beispielsweise an folgenden Zahlen ablesen: Wie vor dreißig Jahren hat der Arbeiter-Samariter-Bund (ASB), bei 1,1 Millionen Mitgliedern, über 11.000 Ehrenamtliche, doch statt über 647 bezahlte Kräfte verfügt er im Jahr 2003 über 16.400 Angestellte und 2.600 Zivildienstleistende. Gleichzeitig stieg die Bilanzsumme innerhalb von 25 Jahren von 39 Millionen Euro auf 982 Millionen Euro. Während das bürgerschaftliche Engagement in absoluten Zahlen stabil blieb, hat es in relativen Zahlen im Verhältnis zur bezahlten Dienstleistungserbringung rapide abgenommen und dabei auch seine Funktionen verändert.

Gleichwohl wäre es verfehlt, Organisationswandel, Organisationsentwicklung und bürgerschaftliches Engagement lediglich unter dem Aspekt einer Zunahme der professionell erbrachten Sozialdienstleistungen zu betrachten. Ein nachhaltig wirkender Bruch stellte das Ende der Kon- kurrenz der Systeme vor bald zwanzig Jahren dar, mit dem sich die politischen Grundlagen und damit die Rahmenbedingungen des wohlfahrtsstaatlichen Arrangements änderten.

Einerseits verflüssigten sich Staats- und Bürgerverständnisse unter dem Eindruck der Rolle zivilgesellschaftlicher Akteure von der Solidarnosc bis zu Vaclav Havel bei der Überwindung des real existierenden Ostblocks. Doch auch im Westen war einiges passiert. Neue soziale Bewegungen und neue zivilgesellschaftliche Aktionsformen hatten die politische und öffentliche Agenda verändert und Umweltschutz, internationale Menschenrechte und eine Modernisierung des Geschlechterverhältnisses in den Blickpunkt öffentlicher Debatten gerückt. Eine Rückbesinnung auf die Potenziale bürgerschaftlicher Selbstorganisation und bürgerschaftlichen Engagements begann, die seitdem nicht nur in Deutschland, sondern in vielen Ländern der Erde auf allen Kontinenten zu politischen und gesetzlichen Verbesserungen geführt haben. Manche Länder sind noch dabei, überhaupt erst rechtliche Grundlagen für bürgerschaftliches Engagement zu schaffen wie beispielsweise China. Andere Länder mit etablierten Strukturen haben zielgerichtet Verbesserungen eingeleitet. In Deutschland widmete man sich seit 1998 mehrfach der Reform des Stiftungsrechts, in Japan wurde 1998 mit einem Gesetz für Non-Profit-Organisationen Raum für eine neue Organisationskultur geschaffen und Frankreich hat 2003 gleich sein gesamtes Vereins-, Spenden- und Stiftungsrecht erheblich reformiert, um privates bürgerschaftliches Engagement zu ermutigen.

Die nur andeutbare internationale Dynamik lässt auch die Einrichtungen und Verbände der Freien Wohlfahrtspflege in Deutschland nicht unberührt. Sie erhalten, als klassische Organisationen, die aus privatem, bürgerschaftlichem Engage- 
ment hervorgegangen sind, durch diesen Prozess potenziell eine neue, verbesserte Legitimität - und zwar unabhängig davon, wie effizient sie soziale Dienstleistungen erbringen können! Dieser Vorteil geht aber einher mit einem kritischeren Blick auf die Freie Wohlfahrtspflege. Inwieweit ist sie tatsächlich noch ein Ort, in dem bürgerschaftliches Engagement in relevanter Weise den Alltag und das alltägliche Geschäft prägen? Die EnqueteKommission Zukunft des bürgerschaftlichen Engagements des Deutschen Bundestages hatte 2002 ihre Zweifel erkennen lassen und einen wesentlichen Teil ihres Berichtes und ihrer Empfehlungen der Organisationsentwicklung der vorhandenen zivilgesellschaftlichen Einrichtungen gewidmet.

Das Ende der Systemkonkurrenz in den 1980er Jahren hatte aber nicht nur die skizzierte zivilgesellschaftliche Dimension. Sie wurde und wird auch als Beweis der Überlegenheit »des Marktes « interpretiert - eine kühne These, wenn man bedenkt, dass die in der Systemkonkurrenz der DDR überlegene Bundesrepublik eine Staatsquote von gut 50 Prozent aufwies. Der Ausbau des Sozialstaates hatte als ein wichtiges politisches Motiv gehabt, beweisen zu wollen, dass der Weg der wohlfahrtsstaatlichen und ordnungspolitischen Regulierung kapitalistischer Marktwirtschaft besser ist als dessen Ersetzung durch einen diktatorischen Staatssozialismus. Seit dem Ende der Systemkonkurrenz gelten »der Markt « und die globalisierte Konkurrenz hingegen nicht mehr zuerst als ein zu regulierendes und einzuhegendes Problem, sondern als Quelle der Lösung aller Probleme.

\section{Neue Weltordnung und die Folgen für die Wohlfahrtsverbände}

Der eigentliche Paradigmenwandel ist tiefgründiger als das kaum noch ernst zu nehmende Gerede über »Neoliberalismus «. Er besteht in einem Klimawandel anderer Art, einer fast sakralen Orientierung an Markt- und Konkurrenzlogik als heiligem Weg zu Effizienz und Effektivität: Vor dem Blick auf das Wetter folgt unvermeidlich die Deutung der Börse und Leiter von Wirtschaftsforschungsinstituten mutieren zu Weisen mit seherischen Gaben.
Das hat für die Freie Wohlfahrtspflege gleich mehrere Konsequenzen. Im Zuge des Rückbaus sozialstaatlicher Leistungen wächst ihre Klientel, bekommt sie potenziell und real mehr zu tun. Dadurch werden die Akteure der Freien Wohlfahrtspflege verstärkt als Dienstleister, zugleich aber auch als Themenanwälte gefordert. Gleichzeitig jedoch müssen sie sich einem Ökonomisierungsdruck stellen, der weit über die Frage nach wirtschaftlicher Vernunft hinaus ökonomische Effizienz und Effektivität zu fraglosen Zielen macht.

Die neue Würde der Konkurrenzlogik des Marktes und das neue Interesse an der Zivil- oder Bürgergesellschaft bestehen zumeist ohne Berührung nebeneinander, wobei dem Markt die höhere öffentliche und politische Aufmerksamkeit gewidmet wird. Das gilt auch für die Freie Wohlfahrtspflege. Manchmal aber werden beide Stränge verknüpft, wenn beispielsweise die Europäische Kommission als Hüter des Marktes ins Spiel kommt. Auf europäischer Ebene versuchen die Wohlfahrtsverbände Eingriffe in ihr Wirtschaften abzuwehren, indem sie sich als zentralen Ort der Organisation und Förderung des bürgerschaftlichen Engagements darstellen. Im Unterschied $\mathrm{zu}$ privatnützigen Profitbetrieben würden sie Sozialkapital und »zivilgesellschaftlichen Mehrwert « herstellen. Sie versuchen damit an die auch von der Enquete-Kommission Zukunft des bürgerschaftlichen Engagements vertretene These anzuschließen, nach der die Bildung von Sozialkapital eine besondere Leistung von zivilgesellschaftlichen Organisationen sei, die weder vom Staat noch von der Erwerbswirtschaft erbracht werden könne und die für das Funktionieren von Staat und Wirtschaft unabdingbare Voraussetzung sei.

Die Herstellung dieses »zivilgesellschaftlichen Mehrwertes « ist auf dem Papier allerdings leichter darstellbar als im Alltag. Die Wiederentdeckung zivilgesellschaftlicher Selbstorganisation und die paradigmatische Sakralisierung des Marktes stellen für die Einrichtungen und Verbände der Freien Wohlfahrtspflege einander widersprechende externe Anforderungen dar, die nicht ohne weitere Anstrengung integrierbar sind.

Die Alternative, vor denen die Einrichtungen dabei stehen, werden im Folgenden als Gegensatz zwischen einem Orga- nisationswandel im Zeichen von Ökonomisierung und Organisationsentwicklung in Verbindung mit bürgerschaftlichem Engagement diskutiert.

\section{Organisationswandel im Zeichen der Ökonomisierung}

Organisationswandel bezeichnet einen ständigen Vorgang, der zumeist unbemerkt verläuft, als Ergebnis vieler, häufig unscheinbarer Variablen, die innerhalb oder außerhalb der Organisation angesiedelt sind. Im Bewusstsein der Akteure erscheinen Entscheidungen im Kontext von Organisationswandel stets notwendig und unvermeidbar zu sein. Altkanzler Helmut Schmidt hatte das Verdienst, dafür den Begriff des »Sachzwangs « zu popularisieren.

Organisationswandel im Zeichen der Ökonomisierung ist rational, da es sich um eine Anpassung an eine vorhandene und verteilungsmächtige gesellschaftliche Grundströmung handelt. Solch eine Anpassung ist ein verantwortliches Handeln, wenn es gelingt, die angemessene und würdige Bezahlung und Beschäftigung der hauptamtlichen Mitarbeiterinnen und Mitarbeiter der Einrichtungen so zu sichern. Diese Anpassung hat im Wohlfahrtsbereich - wie im deutschen NonProfit-Bereich insgesamt - fünf Hauptpfade genommen:

- Improvisationen am Rande der Legalität,

- die kooperative Bejahung von Leistungsverträgen,

- die Ausgründung von Einrichtungen und

- die Rekomposition etwa in Form einer Holding oder unter dem Dach einer gemeinnützigen Aktiengesellschaft.

- Wie Neumann zeigte, gibt es noch das Prinzip der Überwinterung: Man tut gegenüber Zuwendungs- und Geldgebern nur so, als ob man sich betriebswirtschaftlich effizienter aufstellt und an veränderte ökonomische und politische Rahmenbedingungen anpasst.

Jeder dieser Pfade hat unmittelbar sein eigenes Risiko, etwa das Abgleiten in Rechtsbeugung und Illegalität beim Improvisieren am Rande der Legalität oder die Entdeckung von Pseudoanpassung durch Zuwendungsgeber bei der »Überwinterung «, was zu übermäßigem Mittelentzug führen kann. Leistungsverträge 
und Ausgründungen gehören zu hauptsächlich verfolgten Wegen. Ihnen ist als Problem inhärent, zu einem Nebeneinander von dienstleistungsorientierten Einrichtungen und bürgerschaftlichem Engagement zu führen, wenn sie als Antwort auf Ökonomisierungsdruck konzipiert werden.

Das eigentliche Problem eines Organisationswandels im Zeichen der Ökonomisierung ist gerade in ökonomischer Hinsicht grundsätzlicher Natur. In gängigen ökonomischen Theorien über die Existenz des Non-Profit-Bereichs, zu dem die Freie Wohlfahrtspflege in der Regel zu rechnen ist, werden konstitutive Unterschiede zwischen Non-Profit- und Profitsektor festgestellt, die man als Grenzen verstehen kann, jenseits derer ein schädliches Zuviel an ökonomischer Rationalität beginnt.

Nach der Heterogenitätstheorie entstehen Non-Profit-Organisationen, weil Markt und Staat versagen. Weil sich die Politik am medianen Wähler orientiert, werden öffentliche Güter für die Befriedigung von Bedürfnissen jenseits dieses »mittleren " Wählers nicht oder nur unzureichend bereitgestellt. Der Markt wiederum versagt analog, wenn die potenziellen Kunden für die Befriedigung ihrer Bedürfnisse nicht über die nötigen Ressourcen verfügen.

Damit ist die Stunde der Non-ProfitOrganisationen da, die in diese Lücke stoßen. In Erklärungen für deren Bestehen, die auf Vertragsversagen als Ursache abzielen, geht es insbesondere um Güter und Dienstleistungen, bei denen eine Informationsasymmetrie zugunsten des Anbieters, beispielsweise in Pflegeeinrichtungen besteht. Hier hat das Fehlen eines eigensüchtigen Gewinnmotivs einen legitimen Vertrauensvorschuss für die Non-Profit-Organisation zur Folge. Warum, so der Kunde, sollte mich die andere Seite übervorteilen wollen, wenn sie doch selbst davon nichts hat?

In der Interdependenztheorie wiederum stellen Non-Profit-Organisationen zuallererst Reaktionen auf Marktversagen dar. Aufgrund geringerer Transaktionskosten und leichterer Abschaffbarkeit sind sie eine Art Pfadfinder staatlicher Intervention.

Nimmt man solche Erklärungsansätze Ernst, macht die Orientierung an privatwirtschaftlichen, gewinnorientierten Unternehmen keinen Sinn, hängt doch die Rationalität solcher Organisationen an Marktbedingungen, denen ein inhärentes Versagenspotenzial als Ursache für die Existenz der Non-Profit-Organisationen zu eigen ist. Eine solche Orientierung wäre für eine Non-Profit-Organisation eine Orientierung am Versagen.

Was sich in dieser Allgemeinheit abstrakt anhört, schlägt sich im alltäglichen Funktionieren einer solchen Organisation nieder. Gewinnorientierte Privatunternehmen können und wollen gar nicht verhindern, dass das Prinzip der Gewinnop-

ihre Tradition Rücksicht genommen. Insbesondere in der Freien Wohlfahrtspflege bedeutet dies das Austarieren durchaus unterschiedlicher Interessenlagen von Haupt- und Ehrenamtlichen in der eigenen Organisation. Hinzu kommen noch weitere Akteure des bürgerschaftlichen Engagements, die berechtigte Ansprüche als Beteiligte an der Organisation anmelden können, beispielsweise Mitglieder und Spender.

Eine bedeutende Frage spielt bei dem Thema Organisationsentwicklung und

\section{»Eine alleinige Orientierung am Markt wäre für eine Non-Profit-Organisation eine Orientierung am Versagen"}

timierung oder Gewinnmaximierung ein zentrales Anreizsystem bei der internen Personalführung ist. »Leistung « soll sich finanziell und bei der Karriere lohnen, bis hin zu nach oben offenen exorbitanten Gehältern und Prämien. Und auch wenn jede Managementlehre im Profitbereich intrinsische Motive im Interesse der Gewinnoptimierung mobilisieren will, wird eines nicht aus dieser Art von Unternehmung: ein wertegebundener Tendenzbetrieb.

\section{Organisationsentwicklung und bürgerschaftliches Engagement}

Organisationsentwicklung bezeichnet einen spezifischen, aktiven Umgang mit Organisationswandel, bei dem in besonderer Weise partizipative und demokratische Vorstellungen berücksichtigt werden. Dabei "wird als essenziell angesehen, dass die Betroffenen von Anfang an in organisatorische Veränderungs- und Anpassungsprozesse eingeschaltet werden und diese in allen Phasen aktiv mitgestalten können (>Betroffene zu Beteiligten machen $<)$ «. Organisationsentwicklung hat zur Voraussetzung, dass es bei den organisationalen Entscheidungen mindestens die echte Wahl zwischen zwei Optionen gibt. Im Prozess wird durch Partizipation auf die Dimension der Wertgebundenheit der Organisation und bürgerschaftliches Engagement, welche Rolle die identifizierbaren Destinatäre oder Nutznießer von Leistungen im Verständnis von gemeinnützigen Dienstleistungsorganisationen haben. Sieht man in ihnen Bedürftige und Schutzbefohlene, wird man bürgerschaftliches Engagement bestenfalls noch als ein didaktisches Mittel ansehen, um einzelnen Personen zu größerer Selbstständigkeit zu verhelfen. Im Zentrum des Verhältnisses stehen Kategorien wie Schutz und Betreuung.

Die Idee »Kunde « hingegen führt, wie bei dem Leitbild der »Dienstleistungskommune", zu einer ökonomistischen Interpretation des Verhältnisses, bei dem die »Kunden « durch eine Art Non-ProfitKaufhaus laufen. Ein Ort für bürgerschaftliches Engagement ist hier nicht zu sehen: Selbst der Weihnachtsmann arbeitet im Kaufhaus nur, wenn er bezahlt wird. Die Kundenidee korreliert am besten mit dem im vorigen Abschnitt diskutierten Organisationswandel unter dem Primat ökonomischer Effizienz- und Effektivitätskriterien.

Eine weitere Idee kann, in Analogie zum Leitbild der Bürgerkommune, die des (heranwachsenden) Bürgers sein. Diese Bürger sind in der einen oder anderen Weise auf die Dienstleistungen der NonProfit-Organisation angewiesen: als Eltern darauf, dass im freigemeinnützigen Kindergarten eine gute und liebevolle Förderung der Kinder stattfindet oder in vielen Notsituationen darauf, dass die 
Selbstachtung und die Achtung durch andere als Bürger nicht verloren gehen.

Die Bürgeridee schließt weder aus, dass man auch ein Kunde ist, der Anspruch auf freundliche Behandlung hat, oder einfach nur Schutzbedürftiger ist, der Hilfe benötigt. Mit der Idee » Bürger « ist in diesem Kontext der Bezug zur Bürgerschaft, also die politisch-öffentliche Selbstorganisation unseres Gemeinwesens und der aus dieser Teilhabe folgenden Rechte und Entwicklungsmöglichkeiten mitgedacht. Bürgerschaftliches Engagement ist hierbei ein kongeniales Alter Ego, das eine NonProfit-Organisation von allen Seiten her durchziehen kann. Aus Hilfebedürftigen werden vielleicht Spender oder Ehrenamtliche, die mitreden und mitgestalten, aus Ehrenamtlichen Hauptamtliche und umgekehrt, und vielleicht benötigt der ein oder andere von ihnen dann selber wieder die Unterstützung und Hilfe der NonProfit-Organisation.

Betrachtet man die Einrichtungen der Freien Wohlfahrtspflege als ein zusammenhängendes Netz, so ist für viele Menschen dieses Modell bereits eine selbstverständliche Realität: Einerseits nehmen sie bestimmte Leistungen sozialer Dienste wahr, andererseits sind sie in anderen Einrichtungen haupt- und ehrenamtlich tätig - und an Weihnachten oder bei Unglücksfällen spenden sie noch für gute Zwecke.

Hat aber nicht das eingangs gegebene Beispiel des Arbeiter-Samariter-Bundes gezeigt, dass ein am Ökonomisierungsdruck orientierter Organisationswandel im Interesse des bezahlten Personals unausweichlich ist und zu einer Aushöhlung von innen führen muss? Gibt es Besonderheiten der internen Steuerung von Karrierewegen im Non-Profit-Sektor und einer von Staat und gewinnorientierter Wirtschaft abweichenden Logik der Rekrutierung von Personal?

Frantz konnte im Rahmen einer Untersuchung von Karrierewegen am Beispiel deutscher Nichtregierungsorganisationen (NRO) zeigen, dass in fast allen Fällen die Hauptamtlichen aus Familien kamen, in denen schon intensive Beziehungen zu sozialen Bewegungen in den jeweiligen Arbeitsfeldern bestanden. So banal diese Einsicht in frühe kindliche Prägungen auch scheint, ist sie alles andere als trivial, da sie auf eine hohe und intensive Wertbindung und ideologische Fundierung der Tätigkeit der Hauptamtlichen hinweist.
Das wird durch eine großangelegte Befragung von ehren- und hauptamtlichen Führungskräften in Non-Profit-Organisationen bestätigt, in dessen Rahmen 2.040 Interviews durchgeführt wurden. Dabei zeigte sich sogar im Vergleich der ehrenund hauptamtlichen Führungskräfte, dass die hauptamtlichen Akteure über eine dichtere und intensivere Engagementbiografie verfügen, als die ehrenamtlichen Führungskräfte. In allen Altersgruppen ist es so, dass die hauptamtlichen Führungskräfte in einem deutlich höheren Prozentsatz schon zuvor ehrenamtlich engagiert waren, als die ehrenamtlichen Führungskräfte. Bei den unter 30jährigen hauptamtlichen Führungskräften waren 62,5 Prozent vorher schon ehrenamtlich verantwortlich in Non-Profit-Organisationen tätig gewesen, aber nur 51,9 Prozent der ehrenamtlichen Führungskräfte. Bei der Altersgruppe ab 51 Jahre sind 76,1 Prozent der hauptamtlichen und 70,6 Prozent der ehrenamtlichen Führungskräfte zuvor schon ehrenamtlich in NonProfit-Organisationen tätig gewesen. Die dichtere Engagementbiografie wird von 60 Prozent der hauptamtlichen Führungskräfte als wichtige Voraussetzung ihrer aktuellen Tätigkeit bejaht. Die Autoren der Studie resümieren diesen Aspekt so: »An diesen Ergebnissen wird auch aus inhaltlicher Perspektive der Stellenwert des Ehrenamts als berufliche Sozialisationsinstanz für gemeinnützige Organisationen ... erkennbar.«

Am Status, den Non-Profit-Organisationen dem bürgerschaftlichen Engagement gegenwärtig und künftig zuweisen, hängt also bedeutend mehr als nur die Frage, wie man eventuell mehr Geld- und Zeitspenden einwerben kann. Es geht

- um die Erzeugung zivilgesellschaftlichen Mehrwerts (gesellschaftliche Basisfunktion),

- um den Bürgerstatus der Nutznießer (Selbstverständnis),

- um die Aufgabe Marktversagen zu kompensieren (ökonomische Funktion),

- um die Aufgabe Staatsversagen zu kompensieren und $\mathrm{zu}$ thematisieren (politische Funktion)

- und um die Sozialisation des eigenen hauptamtlichen Personals (arbeitsweltliche Funktion).

Mit anderen und resümierenden Worten: Bürgerschaftliches Engagement ist nicht ein Zusatz oder ein Randthema der
Organisationsentwicklung von Einrichtungen und Verbänden der Freien Wohlfahrtspflege, sondern führt ins Zentrum der Zukunftsfähigkeit als gemeinnütziger und wertegebundener Einrichtung.

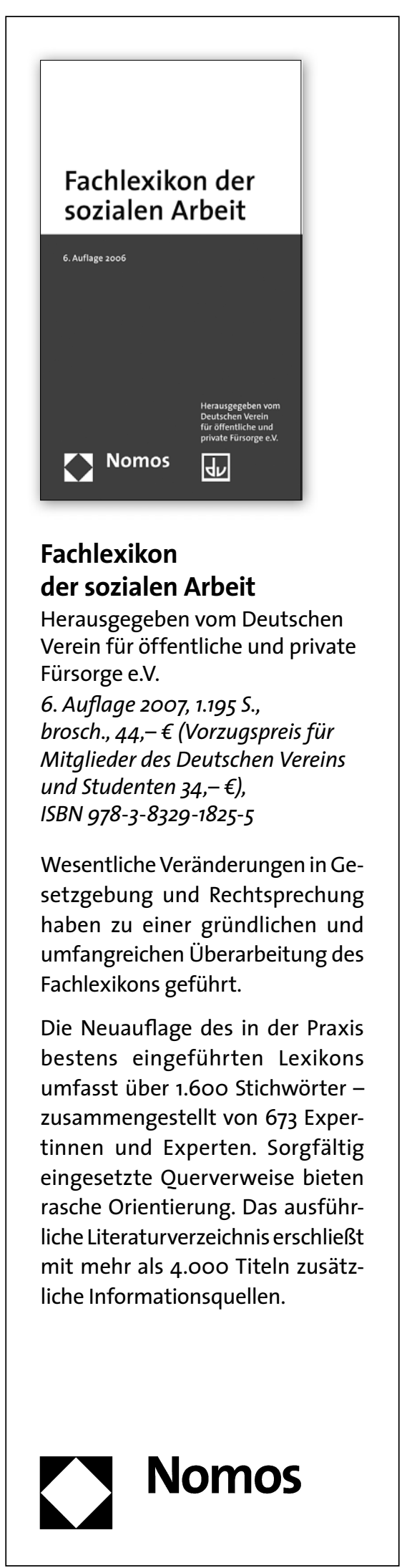

